\title{
Reduced-Complexity Estimation for Large-Scale Hidden Markov Models
}

\author{
Subhrakanti Dey, Member, IEEE, and Iven Mareels, Fellow, IEEE
}

\begin{abstract}
In this paper, we address the problem of reduced-complexity estimation of general large-scale hidden Markov models (HMMs) with underlying nearly completely decomposable discrete-time Markov chains and finite-state outputs. An algorithm is presented that computes $O(\epsilon)$ (where $\epsilon$ is the related weak coupling parameter) approximations to the aggregate and full-order filtered estimates with substantial computational savings. These savings are shown to be quite large when the chains have blocks with small individual dimensions. Some simulation studies are presented to demonstrate the performance of the algorithm.
\end{abstract}

Index Terms-Computational complexity, hidden Markov models, Markov chains, nearly completely decomposable, state estimation.

\section{INTRODUCTION}

$\mathbf{R}$ EDUCING computational complexity in optimal estimation and control of large-scale Markov chains has been a topic of substantial interest [1]-[4]. A class of such Markov chains is also known as "nearly completely decomposable Markov chains." These Markov chains are usually large scale and show strong interactions within groups and weak interactions between the groups. These chains are usually characterized by transition probability matrices that can be expressed as $P=I_{n}+A+\epsilon B$, where $I_{n}+A$ is a block diagonal matrix with the individual blocks being stochastic matrices, the number of blocks being $N$. These blocks are also termed as "superstates" (as in [15]). Typically, $n \gg N$, where $n$ is the total number of states in the chain. The parameter $\epsilon>0$ is small and acts as the a weak coupling parameter that makes the chain "nearly" completely decomposable (the chain is completely decomposable if $\epsilon=0$ ). Applications of such Markov chains to economic modeling, queueing networks, and computer systems have been reported in early works such as [5] and [6]. Other applications can also be found in manufacturing systems operating with machines of varying speed [7], communication networks with variable bit rate video traffic [8], etc. In [1], [2], [9], and [10], the authors focus on obtaining reduced-complexity computation of the stationary distribution of such Markov chains using various aggregation-decomposition methods. The singular perturbation approach to aggregation of finite-state Markov chains has

\footnotetext{
Manuscript received February 20, 2003; revised July 4, 2003. The associate editor coordinating the review of this manuscript and approving it for publication was Prof. Tulay Adali.

The authors are with the Department of Electrical and Electronic Engineering, University of Melbourne, Parkville, 3010, Australia (e-mail: sdey@ee.mu.oz.au).

Digital Object Identifier 10.1109/TSP.2004.826171
}

been studied in [11]-[13], among others. Much of this work concentrated on obtaining reduced-complexity controllers for these Markov chains, very little attention was paid to the case of partially observed nearly completely decomposable Markov chains. Only [3] and, more recently, [4] and [14] address this problem. In [14], weak convergence results for hybrid filtering problems involving nearly completely decomposable hidden Markov chains are presented. The research reported in [3] and [4] partially address the case of reduced-complexity state estimation from imperfect observations but does not provide a systematic way of obtaining reduced-order computations of the filtered estimates that exploits the near-complete decomposability of the Markov chain to arrive at substantial guaranteed computational savings with rigorous performance bounds. The trade-off between accuracy and computational complexity in state estimation of hidden nearly completely decomposable Markov chains is also not explored in [3] or [4].

Computational complexity for filtering of hidden Markov models (HMMs) with underlying nearly completely decomposable Markov chains was first addressed in our own work [15] and the following paper [16], where algorithms were presented for $O\left(\epsilon^{2}\right)$ approximations to the aggregate and full-order state estimates requiring $O\left(N^{2}\right)$ computations per discrete time instant, as opposed to $O\left(n^{2}\right)$ computations for the optimal estimates. These results were proven using ergodicity assumptions on the HMMs and for sufficiently small $\epsilon$. However, a restriction was imposed on the state-to-output observation probability matrices in that they were assumed to have a "block" structure. This implied that the output transitions only depended on the superstates but did not distinguish among the states belonging to the same superstate. It was shown in [15] how this assumption allowed us to obtain a nice decoupling transformation that is uniformly bounded and results in $O\left(\epsilon^{2}\right)$ approximations to the aggregate and full-order filtered estimates and smoothed estimates [16]. It is perhaps not surprising that this special structure of the state-to-output transition matrix allows one to obtain $O\left(\epsilon^{2}\right)$ approximation, whereas generally speaking, one would expect to obtain $O(\epsilon)$ approximations. Mathematically, this structure helps us to solve for the decoupling transformation that satisfies a nonlinear matrix equation, using iterative approximation techniques (see [15]). These iterative techniques are easily implemented because the nonlinear term in the equation is weighted only by the weak coupling parameter $\epsilon$, due to the special structure on the state-to-output transition matrix. It is also clear that under any small perturbation (which is of any polynomial order in $\epsilon$ ) applied to the state-to-output transition matrix with the special block structure, the methods presented in [15] can be applied 
as well, but not to any general state-to-output transition matrix that does not have a block structure or a nearly block structure.

In this paper, we lift this restriction on the state-to-output transition probability matrix and allow it to be any general transition probability matrix with all positive elements. We concentrate on a discrete-time irreducible nearly completely decomposable Markov chain with finite-state outputs. We provide a systematic way for obtaining reduced-complexity filtered estimates for these HMMs with a prescribed degree of accuracy. The contribution of this work lies in

1) presenting an algorithm that computes guaranteed $O(\epsilon)$ approximations to the aggregate and full-order filtered estimates for a general hidden Markov model with finite-state outputs;

2) showing that these approximations can be calculated with substantial computational savings when the chain has superstates that have small individual dimensions;

3) demonstrating through simulation studies the effectiveness of the algorithm.

The main assumptions that are used involve ergodicity assumptions on the hidden Markov model and that $\epsilon$ is sufficiently small. We should also point out that these results have important implications for reduced-complexity estimation for HMMs with continuous-valued outputs as well. However, extensions of some of the rigorous analysis to the continuous-valued output case are still incomplete and part of our ongoing research.

The rest of the paper is organized as follows. Section II details the signal model that we are interested in and the corresponding technical assumptions. Section III presents the basics on conditional probability-based state estimation for HMMs with a transformation technique that allows recursive calculations of aggregate as well as full-order state estimates and makes the reduction of complexity possible. Section IV presents the main technical assumptions and results on reduced-complexity $O(\epsilon)$ approximate filtering. Section $\mathrm{V}$ presents some simulation studies that demonstrate the performance of our algorithm. Section VI presents some concluding remarks.

\section{SignAl MODEL}

A discrete-time nearly completely decomposable Markov chain $X_{k}$ in a probability space $(\Omega, \mathcal{F}, \mathcal{P})$ comprising $n$ states is characterized by a transition probability matrix $P^{\epsilon} \in \mathbb{R}^{n \times n}$

$$
P^{\epsilon}=I_{n}+A+\epsilon B
$$

where $I_{n}$ is the identity matrix of order $n \times n$, and $A$ is block diagonal with

$$
A=\left[\begin{array}{ccccc}
A_{11} & 0 & . & . & 0 \\
0 & A_{22} & 0 & . & \cdot \\
0 & 0 \cdot & . & . & . \\
. . & . \cdot & . & . & \cdot \\
0 & \cdot & 0 & \cdot & A_{N N}
\end{array}\right]
$$

where $A_{i i} \in \mathbb{R}^{n_{i} \times n_{i}}, \forall i, \sum_{i} n_{i}=n, \epsilon>0$ is a small perturbation parameter, and $B \in \mathbb{R}^{n \times n}$. It is clear that there are $N$ blocks in the Markov chain, within each of which the dynamics is fast, and every so often, the chain leaves one block to visit another. Since $\epsilon$ is small, the rate at which these interblock transitions occur is slow. For all $i, I_{n_{i}}+A_{i i}$ is row-stochastic, and so is $P$. Obviously, the row-sums of $A$ and $B$ are zero. Just as in [15], we make the following natural, key assumption.

Assumption 2.1: The matrices $P^{\epsilon}$ and $I_{n_{i}}+A_{i i}, \forall i$ are irreducible.

Remark 1: Notice that the above Assumption 2.1 guarantees the existence of a unique stationary distribution of $P^{\epsilon}$ and $I_{n_{i}}+$ $A_{i i} \forall i$. There is one difference, however, that should be noted: While the stationary distribution of $P^{\epsilon}$ depends on $\epsilon$, those of $I_{n_{i}}+A_{i i}, i=1,2, \ldots, N$ do not.

The states of the Markov chain are observed through another stochastic process (observation or measurement process) $Y_{k}$. For the analytical development of the paper, we assume that $Y_{k}$ belongs to a discrete set of finite cardinality. More specifically, $Y_{k} \in\{1,2, \ldots, M\}$, and $\mathcal{P}\left(Y_{k}=i \mid X_{k}=j\right)=c_{i j}, i=$ $1,2, \ldots, M, j=1,2, \ldots, n$. Such a signal model (irrespective of whether the underlying Markov chain is nearly completely decomposable or not) is also known as an HMM. Note also that $\sum_{i} c_{i j}=1, \forall j$, that is, the observation probability matrix $C=\left(c_{i j}\right)$ is column-stochastic. We assume that $c_{i j}>0, \forall i, j$.

It was shown in [15] that reduced-order computations $\left[O\left(N^{2}\right)\right.$ at each discrete time instant] for the filtered state estimate (for extension of these results to smoothing, see [16]) can be obtained with $O\left(\epsilon^{2}\right)$ approximation when the state-to-output transition matrix $C$ has a special structure, namely, $c_{i j}=\bar{c}_{i l}, \forall j \in S_{l}, \forall i$. Since, typically, $n \gg N$, these computational savings are substantial. In this paper, we remove this restriction on $C$.

In what follows, we will show that even with no restrictions on $C$, one can obtain $O(\epsilon)$ approximations to the filtered estimates with substantial computational reductions when the individual block sizes $n_{i}, i=1,2, \ldots, N$ are not too large. As an example, one will obtain substantial reduction in computational complexity if $n=200, N=25, n_{i}=8, \forall i$ instead of $n=200, N=4, n_{i}=50, \forall i$. A table consisting of exact numbers of multiplications, additions, and divisions per discrete time instant will be provided later to compare the computational complexity of exact state estimation and the approximate state estimation.

As in [15], we term the $N$ blocks as "superstates." Notice that the probability (or conditional probability) of the Markov chain belonging to a particular superstate is the sum of probabilities (or conditional probabilities) of the chain belonging to its constituent states. We denote the $l$ th superstate by $S_{l}, l=1,2, \ldots, N$. Without loss of generality, $S_{1}=\left\{1,2, \ldots, n_{1}\right\}, S_{2}=\left\{n_{1}+1, n_{1}+2, \ldots, n_{1}+n_{2}\right\}$, etc. We also term (like in [15]) the filtered estimate vector with lth element being $P\left(X_{k} \in S_{l} \mid \mathcal{Y}_{k}\right)$ as the aggregate filtered estimate $l=1,2, \ldots, N$. Here, $\mathcal{Y}_{k}$ is the complete filtration generated by the $\sigma$ algebra $\sigma\left(Y_{0}, Y_{1}, \ldots, Y_{k}\right)$.

\section{STATE ESTIMATION OF HMMs}

It is well known that the conditional filtered state estimate for an HMM is defined in the following way:

$$
\alpha_{k}(i)=\mathcal{P}\left(X_{k}=i \mid \mathcal{Y}_{k}\right)
$$


where $\mathcal{Y}_{k}$ is the complete filtration generated by the $\sigma$ algebra $\sigma\left(Y_{0}, Y_{1}, \ldots, Y_{k}\right)$. Defining the row vector $\alpha_{k} \triangleq\left(\alpha_{k}(1) \alpha_{k}(2)\right.$ $\left.\ldots \alpha_{k}(n)\right)$, one can obtain the following recursion [17]:

$$
\begin{aligned}
\alpha_{k+1} & =\frac{1}{Z_{k+1}} \alpha_{k} P^{\epsilon} C\left(Y_{k+1}\right) \\
\alpha_{0} & =\pi_{0} C\left(Y_{0}\right)
\end{aligned}
$$

where $C\left(Y_{k+1}\right)=\operatorname{diag}\left\{c_{i 1} c_{i 2} \ldots c_{i n}\right\}$ if $Y_{k+1}=i$, and $Z_{k+1}=\alpha_{k} P^{\epsilon} C\left(Y_{k+1}\right) 1_{n}$ is the normalization factor (with $1_{n}$ being the $n$-length column vector of all 1-s), and $\pi_{0}$ is the row vector representing the initial distribution of $X_{0}$.

Note that the aggregate filtered state estimate is given by

$$
\zeta_{k}(j)=\mathcal{P}\left(X_{k} \in S_{j} \mid \mathcal{Y}_{k}\right)=\sum_{l \in S_{j}} \mathcal{P}\left(X_{k}=l \mid \mathcal{Y}_{k}\right)
$$

Obviously, the row vector $\zeta_{k} \triangleq\left(\zeta_{k}(1) \zeta_{k}(2) \ldots \zeta_{k}(N)\right)$ denotes the aggregate filtered state estimates and can be represented by

$$
\zeta_{k}=\alpha_{k} W_{1}
$$

where $W_{1} \in \mathbb{R}^{n \times N}$ is given by

$$
W_{1}=\left[\begin{array}{cccc}
1_{n_{1}} & 0 & 0 & 0 \\
0 & 1_{n_{2}} & \cdot & \cdot \\
\cdot & 0 & \cdot & 0 \\
0 & \cdot & \cdot & 1_{n_{N}}
\end{array}\right] .
$$

Following the same techniques as in [10] (also used in [15]), another matrix $W_{2} \in \mathbb{R}^{n \times(n-N)}$ is chosen such that the transformation $\Gamma=\left[\begin{array}{ll}W_{1} & W_{2}\end{array}\right]$ is nonsingular. Let $\eta_{k}=\alpha_{k} W_{2}$. In addition, let $\Gamma^{-1}=\left[\begin{array}{l}V_{1} \\ V_{2}\end{array}\right]$, where obviously, $V_{1} \in \mathbb{R}^{N \times n}$, and $V_{2} \in \mathbb{R}^{(n-N) \times n}$. As in [15], which is motivated to make further computations simple, we choose the $i$ th diagonal block in $W_{2}$, namely, $W_{2}^{(i)}\left(\in \mathbb{R}^{n_{i} \times n_{i}-1}\right)$, to be

$$
W_{2}^{(i)}=\left[\begin{array}{c}
0 \cdots 0 \\
I_{n_{i}-1}
\end{array}\right] \text {. }
$$

For this choice, the $i$ th diagonal blocks of $V_{1}, V_{2}$ turn out to be

$$
\begin{aligned}
& V_{1}^{(i)}=\left[\begin{array}{lll}
1 & 0 & 0 \ldots 0
\end{array}\right] \\
& V_{2}^{(i)}=\left[\begin{array}{cc}
-1 & \\
-1 & \\
\cdot & I_{n_{i}-1} \\
-1 &
\end{array}\right]
\end{aligned}
$$

Here, $V_{1}^{(i)} \in \mathbb{R}^{1 \times n_{i}}, V_{2}^{(i)} \in \mathbb{R}^{\left(n_{i}-1\right) \times n_{i}}$.

Now, rewrite (3) as

$$
\begin{aligned}
{\left[\begin{array}{ll}
\zeta_{k+1} & \eta_{k+1}
\end{array}\right] } & =\frac{1}{Z_{k+1}}\left[\begin{array}{ll}
\zeta_{k} & \eta_{k}
\end{array}\right]\left[\begin{array}{c}
V_{1} \\
V_{2}
\end{array}\right] P^{\epsilon} C\left(Y_{k+1}\right)\left[W_{1}\right. \\
& =\frac{1}{Z_{k+1}}\left[\begin{array}{ll}
\zeta_{k} & \eta_{k}
\end{array}\right]\left[\begin{array}{ll}
\tilde{A}_{11}^{k} & \tilde{A}_{12}^{k} \\
\tilde{A}_{21}^{k} & \tilde{A}_{22}^{k}
\end{array}\right]
\end{aligned}
$$

where $\tilde{A}_{11}^{k}=\tilde{A}_{1}^{k}+\epsilon \tilde{B}_{1}^{k}, \tilde{A}_{12}^{k}=\tilde{A}_{2}^{k}+\epsilon \tilde{B}_{2}^{k}, \tilde{A}_{21}^{k}=\tilde{C}_{1}^{k}+$ $\epsilon \tilde{D}_{1}^{k}, \tilde{A}_{22}^{k}=\tilde{C}_{2}^{k}+\epsilon \tilde{D}_{2}^{k}$, and they are given by the following equations:

$$
\begin{array}{ll}
\tilde{A}_{1}^{k}=V_{1}\left(I_{n}+A\right) C\left(Y_{k+1}\right) W_{1}, & \tilde{B}_{1}^{k}=V_{1} B C\left(Y_{k+1}\right) W_{1} \\
\tilde{A}_{2}^{k}=V_{1}\left(I_{n}+A\right) C\left(Y_{k+1}\right) W_{2}, & \tilde{B}_{2}^{k}=V_{1} B C\left(Y_{k+1}\right) W_{2} \\
\tilde{C}_{1}^{k}=V_{2}\left(I_{n}+A\right) C\left(Y_{k+1}\right) W_{1}, & \tilde{D}_{1}^{k}=V_{2} B C\left(Y_{k+1}\right) W_{1} \\
\tilde{C}_{2}^{k}=V_{2}\left(I_{n}+A\right) C\left(Y_{k+1}\right) W_{2}, & \tilde{D}_{2}^{k}=V_{2} B C\left(Y_{k+1}\right) W_{2} .
\end{array}
$$

Clearly, (8) can be carried out in two steps $(\forall k)$ :

Step 1) Calculate the unnormalized quantities $\zeta_{k+1}^{u}, \eta_{k+1}^{u}$ according to the following recursion:

$$
\left[\begin{array}{ll}
\zeta_{k+1}^{u} & \eta_{k+1}^{u}
\end{array}\right]=\left[\begin{array}{ll}
\zeta_{k} & \eta_{k}
\end{array}\right]\left[\begin{array}{ll}
\tilde{A}_{11}^{k} & \tilde{A}_{12}^{k} \\
\tilde{A}_{21}^{k} & \tilde{A}_{22}^{k}
\end{array}\right]
$$

where $\zeta_{0}=\alpha_{0} W_{1}, \eta_{0}=\alpha_{0} W_{2}$.

Step 2) Normalize $\zeta_{k+1}^{u}, \eta_{k+1}^{u}$ by the normalization factor $Z_{k+1}=\sum_{j=1}^{N} \zeta_{k+1}^{u}(j)$. Note also that

$$
\alpha_{k}=\zeta_{k} V_{1}+\eta_{k} V_{2} \text {. }
$$

As observed in [15], we are reminded again that for the above choices of $V_{1}, V_{2}, W_{1}, W_{2}$, the matrices $\widetilde{A}_{1}^{k}, \tilde{A}_{2}^{k}, \tilde{C}_{1}^{k}$ and $\tilde{C}_{2}^{k}$ are block diagonal matrices for all $k$; more specifically, $\tilde{A}_{1}^{k}$ is diagonal, $\tilde{A}_{2}^{k}$ is block diagonal with the $i$ th block being a row vector of size $n_{i}-1, \tilde{C}_{1}^{k}$ is block diagonal with the $i$ th block being a column vector of size $n_{i}-1$, and $\tilde{C}_{2}^{k}$ is block diagonal with the $i$ th block being a square matrix of size $\left(n_{i}-1\right) \times\left(n_{i}-1\right)$. In addition, since the matrices $\tilde{A}_{1}^{k}, \tilde{A}_{2}^{k}, \tilde{C}_{1}^{k}$ and $\tilde{C}_{2}^{k}$ depend only on $Y_{k+1}$, which is finitely valued, one can essentially precompute the matrices $\tilde{A}_{1}^{k}, \tilde{A}_{2}^{k}, \tilde{C}_{1}^{k}$ and $\tilde{C}_{2}^{k}$ for each possible value of $Y_{k+1}$ and store them in a lookup table. During the filtering operations, as and when we get a specific observation, we can obtain the corresponding matrices by table lookup.

\section{IV. $O(\epsilon)$ APPROXIMATE REDUCED-ORDER FILTERS}

In this section, we are concerned with obtaining reducedcomplexity aggregate and full-order filters with a prescribed degree of accuracy when $\epsilon$ is "sufficiently" small. Although the techniques used in this section are similar to that of [15], the results are substantially different due to the absence of a special structure on the state-to-output transition probability matrix $C$, as assumed in [15].

Following the approach in [15], we use a standard decoupling technique to obtain the transformed variables $\left[\begin{array}{ll}\bar{\zeta}_{k} & \bar{\eta}_{k}\end{array}\right]$ that are given by

$$
\left[\begin{array}{ll}
\bar{\zeta}_{k} & \bar{\eta}_{k}
\end{array}\right]=\left[\begin{array}{ll}
\zeta_{k} & \eta_{k}
\end{array}\right]\left[\begin{array}{cc}
I_{N} & L_{k} \\
0 & I_{n-N}
\end{array}\right] .
$$

This also implies that

$$
\left[\begin{array}{ll}
\zeta_{k} & \eta_{k}
\end{array}\right]=\left[\begin{array}{ll}
\bar{\zeta}_{k} & \bar{\eta}_{k}
\end{array}\right]\left[\begin{array}{cc}
I_{N} & -L_{k} \\
0 & I_{n-N}
\end{array}\right] .
$$

Note that one can relate the unnormalized versions of $\bar{\zeta}_{k}, \bar{\eta}_{k}$, which is denoted by $\bar{\zeta}_{k}^{u}, \bar{\eta}_{k}^{u}$, respectively, by the same decou- 
pling transformation (since the normalization factor is the same):

$$
\left[\begin{array}{ll}
\zeta_{k}^{u} & \eta_{k}^{u}
\end{array}\right]=\left[\begin{array}{ll}
\bar{\zeta}_{k}^{u} & \bar{\eta}_{k}^{u}
\end{array}\right]\left[\begin{array}{cc}
I_{N} & -L_{k} \\
0 & I_{n-N}
\end{array}\right] .
$$

Here, $\left\{L_{k} \in \mathbb{R}^{N \times(n-N)}\right\}$ is assumed to be a sequence of uniformly bounded time-varying matrices to be solved for. Using this, together with (8), one can obtain the following recursion in the transformed variables:

$$
\begin{aligned}
{\left[\begin{array}{ll}
\bar{\zeta}_{k+1} \quad \bar{\eta}_{k+1}
\end{array}\right]=} & \frac{1}{Z_{k+1}}\left[\begin{array}{ll}
\bar{\zeta}_{k} & \bar{\eta}_{k}
\end{array}\right] \\
& \times\left[\begin{array}{cc}
\tilde{A}_{11}^{k}-\underset{\tilde{A}_{21}^{k}}{L_{k}} \tilde{A}_{21}^{k} & 0 \\
\tilde{A}_{21}^{k} L_{k+1}+\tilde{A}_{22}^{k}
\end{array}\right]
\end{aligned}
$$

where $L_{k}$ was chosen such that the upper right-hand element of the right-hand side of (15) is zero, or

$$
\left(\tilde{A}_{11}^{k}-L_{k} \tilde{A}_{21}^{k}\right) L_{k+1}=L_{k} \tilde{A}_{22}^{k}-\tilde{A}_{12}^{k}, \quad L_{0}=0
$$

Note that one can recursively solve for $L_{k}$ from the above equation, provided $\tilde{A}_{11}^{k}-L_{k} \tilde{A}_{21}^{k}$ is invertible for every $k$. This involves multiplications of matrices that are not necessarily sparse at each $k$ and requires a large number of computations. A wellknown technique [18] is to exploit the fact that $\epsilon>0$ is a small positive number and truncate a power series expansion of $L_{k}$ in $\epsilon$ at some finite power. However, this approximation is only valid if $L_{k}$ is uniformly bounded.

In [15], the uniform boundedness of $\left\{L_{k}\right\}$ was shown using the irreducibility condition stated in Assumption (2.1) and the special structure assumed on $C$ (see [15] for details) and a number of inequalities that were jointly sufficient to guarantee that $\epsilon$ is sufficiently small. The proof in [15] crucially depended on the special structure of $C$, which implied that the nonlinear term in (16) was of $O(\epsilon)\left(C_{1}^{k}=0\right)$.

Due to the generalized nature of $C$ in the current work, we do not have this convenience. We proceed along a different path to establish the boundedness of $\left\{L_{k}\right\}$ for a sufficiently small $\epsilon$. We first make an assumption that guarantees the existence of a uniformly bounded $L_{k}(0)$, which is the solution to (16) when $\epsilon=0(\forall k)$. Then, we argue that since $L_{k}$, as a solution of (16), is a continuous function of $\epsilon$ in a small neighborhood of $\epsilon=0, L_{k}$ will also be uniformly bounded for a sufficiently small $\epsilon$.

Notice that $L_{k}(0)$ satisfies the following recursion (see also (9)):

$$
\begin{aligned}
L_{k+1}(0) & =\left(\tilde{A}_{1}^{k}-L_{k}(0) \tilde{C}_{1}^{k}\right)^{-1}\left(L_{k}(0) \tilde{C}_{2}^{k}-\tilde{A}_{2}^{k}\right) \\
L_{0}(0) & =0
\end{aligned}
$$

The difference with [15] is that $\tilde{C}_{1}^{k} \neq 0$ in the current situation. Observing that $\tilde{A}_{1}^{k}$ is diagonal and nonsingular, one can rewrite (17) as the following:

$$
\begin{aligned}
L_{k+1}(0)= & \left(\tilde{A}_{1}^{k}\right)^{-1} L_{k}(0) \tilde{C}_{2}^{k}-\left(\tilde{A}_{1}^{k}\right)^{-1} \tilde{A}_{2}^{k} \\
& +\left[\left(\tilde{A}_{1}^{k}\right)^{-1} L_{k}(0) \tilde{C}_{1}^{k} L_{k+1}(0)\right], \quad L_{0}(0)=0 .
\end{aligned}
$$

Below, we use the vec notation associated with a matrix $(\epsilon$ $\left.\mathbb{R}^{m \times n}\right) A=\left[A_{* 1} A_{* 2} \ldots A_{* n}\right]$, which is defined as

$$
\operatorname{vec}(A)=\left[\begin{array}{c}
A_{* 1} \\
A_{* 2} \\
\vdots \\
A_{* n}
\end{array}\right]
$$

where $A_{* i}$ denotes the $i$ th column of the matrix $A$. We also use the $\otimes$ notation to denote the Kronecker product of two matrices. Using the notations $l_{k}^{\prime}=$ vec $\left(L_{k}(0)^{\prime}\right), m_{0}^{\prime}=-\operatorname{vec}\left(\left(\left(\tilde{A}_{1}^{0}\right)^{-1} \tilde{A}_{2}^{0}\right)^{\prime}\right)$ and $g_{k}^{\prime}\left(L_{k-1}(0), L_{k}(0)\right)$ $=\operatorname{vec}\left(\left(-\left(\tilde{A}_{1}^{k}\right)^{-1} \tilde{A}_{2}^{k}+\left(\tilde{A}_{1}^{k}\right)^{-1} L_{k-1}(0)^{\prime} C_{1}^{k} L_{k}(0)\right)^{\prime}\right)$, (18) can be rewritten as

$$
\begin{aligned}
l_{k+1} & =l_{k}\left(\left(\tilde{A}_{1}^{k}\right)^{-1} \otimes \tilde{C}_{2}^{k}\right)+g_{k+1}\left(L_{k}(0), L_{k+1}(0)\right) \\
l_{0} & =0
\end{aligned}
$$

Clearly, repeating this recursion, one can write $\forall k \geq 1$

$$
\begin{aligned}
l_{k+1}= & m_{0} \prod_{j=1}^{k} M_{j+1}+\left[\sum_{p=0}^{k-1} g_{k+1-p}\left(L_{k-p}(0), L_{k+1-p}(0)\right)\right. \\
& \left.\times \prod_{q=p-1}^{0} M_{k+1-q}\right], \quad l_{1}=m_{0}
\end{aligned}
$$

where $M_{k+1}=\left(\tilde{A}_{1}^{k}\right)^{-1} \otimes \tilde{C}_{2}^{k}$, and $\prod_{q=-1}^{0} M_{k+1-q}=$ $I_{N \times(n-N)}$.

Let us now introduce the following notations. We denote by $\|\cdot\|_{\infty}$ the $l_{\infty}$ vector norm and by $\||\cdot|\|_{\infty}$ the corresponding induced maximum absolute row sum matrix norm.

In what follows, we will be looking for sufficient conditions such that $L_{k}(0), \forall k$ belongs to a compact set $\mathcal{D} \triangleq\left\{L:\left\|\operatorname{vec}\left(L^{\prime}\right)\right\|_{\infty}<\bar{L}\right\}$. Notice also that using the special structures of $\tilde{A}_{1}^{k}, \tilde{A}_{2}^{k}, \tilde{C}_{1}^{k}$ and the fact that $Y_{k+1}, \forall k$ can only take finitely many values, one can easily obtain the following over bounds $\bar{a}_{1}, \bar{c}_{1}$ such that if $L_{k}(0) \in \mathcal{D}$, then $\forall k,\left\|g_{k+1}\left(L_{k}(0), L_{k+1}(0)\right)\right\|_{\infty}<\bar{g}=$ $\left(\bar{a}_{1}+\bar{c}_{1}\left(\max _{i} n_{i}-1\right) \bar{L}^{2}\right)$.

Denote $\rho\left(Y_{k+1}\right)=\|\| M_{k+1} \|_{\infty}$. Denote $\pi_{Y}$ as the stationary distribution of $Y_{k}$ such that $\pi_{Y}(m)=\sum_{i} P\left(Y_{k}=m \mid X_{k}=\right.$ i) $\pi(i)=\sum_{i} c_{m i} \pi(i)$, where $\pi_{i}$ is the $i$ th component of the stationary distribution of $X_{k}$ such that $\pi P=\pi$. Denote the expectation taken with respect to the stationary distribution $\pi_{Y}$ as $E_{\pi_{Y}}$.

We now make the following assumptions, which are easy to verify and are sufficient to guarantee the uniform boundedness of $L_{k}(0), \forall k$.

Assumption 4.1:

$$
E_{\pi_{Y}}\left(\ln \rho\left(Y_{k+1}\right)\right)=-\bar{\lambda}<0, \forall k
$$

Remark 2: Note that a similar set of assumptions was made (Assumptions 4.1 and 4.2) in [15] to obtain the uniform boundedness of $L_{k}$ for a sufficiently small $\epsilon$. These two assumptions played a crucial role in guaranteeing the uniform boundedness of $L_{k}$ due to the special structure of the $C$ matrix, where the observation probabilities only depended on the superstates and 
were identical for all the states in the same superstate (for each observation). Since, in the current work, we do not assume such a special structure on $C$, we cannot prove a uniform boundedness of $L_{k}$ directly but make Assumption 4.1, which guarantees the uniform boundedness of $L_{k}(0)$. Later, an additional Assumption 4.2 is made to prove that for sufficiently small $\epsilon>0$, one can show the boundedness of $L_{k}$ using continuity arguments.

Observe that Assumption 4.1 guarantees that $\exists$ a $t^{*}$ such that $\forall k \geq t^{*}+1,\|\| \prod_{j=1}^{k} M_{j+1} \|_{\infty}<\exp \left(-\left(t^{*}+1\right) \bar{\lambda}\right)$ (see [15] for more details). Using this result, we now state the following Lemma.

Lemma 4.1: Suppose Assumption 4.1 holds. Suppose also that there exists an $\bar{L}>0$ such that

$$
\left\|m_{0}\right\|_{\infty} \exp \left(-\left(t^{*}+1\right) \bar{\lambda}\right)+\bar{g} \bar{S}+\bar{g} \frac{\exp \left(-\left(t^{*}+1\right) \bar{\lambda}\right)}{1-\exp (-\bar{\lambda})}<\bar{L}
$$

where $\bar{S}=\sum_{p=0}^{t^{*}} \prod_{q=p-1}^{0}\left\|\left|M_{t^{*}-q}\right|\right\|_{\infty}$ is a finite number, and recall that $\bar{g}=\bar{a}_{1}+\bar{c}_{1}\left(\max _{i} n_{i}-1\right) \bar{L}^{2}$. Then, there exists a uniformly bounded solution $L_{k}(0), \forall k$ to (18) such that $L_{k}(0) \in$ $\mathcal{D}$.

Proof: Since the proof of this Lemma is similar to part of the proof of [15, Lemma 4.1], we provide an outline of the proof here. Recall that Assumption 4.1 implies that $\exists$ a $t^{*}$ such that $\forall k \geq t^{*}+1,\left\|\left|\prod_{j=1}^{k} M_{j+1}\right|\right\|_{\infty}<\exp \left(-\left(t^{*}+1\right) \bar{\lambda}\right)$, and recall as well that $\left\|g_{k+1}\left(L_{k}(0), L_{k+1}(0)\right)\right\|_{\infty}<\bar{g}$, where $\bar{g}=$ $\left(\bar{a}_{1}+\bar{c}_{1}\left(\max _{i} n_{i}-1\right) \bar{L}^{2}\right)$.

Now, if one considers (20) after taking the $\|\cdot\|_{\infty}$ norm of both sides, one gets

$$
\left\|l_{k+1}\right\|_{\infty}<\left\|m_{0}\right\|_{\infty} \exp (-k \bar{\lambda})+\bar{g} \sum_{p=0}^{k-1} \prod_{q=p-1}^{0}\left\|\left|M_{t^{*}-q}\right|\right\|_{\infty} .
$$

Defining $\bar{S}=\sum_{p=0}^{t^{*}} \prod_{q=p-1}^{0}\left\|\mid M_{t^{*}-q}\right\|_{\infty}$, one can then use the above-mentioned bounds on $\left\|\left|\prod_{j=1}^{k} M_{j+1}\right|\right\|_{\infty}$ and $\left\|g_{k+1}\left(L_{k}(0), L_{k+1}(0)\right)\right\|_{\infty}$ to obtain $\left(\forall k \geq t^{*}+1\right)$

$\left\|l_{k+1}\right\|_{\infty}<\left\|m_{0}\right\|_{\infty} \exp \left(-\left(t^{*}+1\right) \bar{\lambda}\right)+\bar{g} \bar{S}+\bar{g} \frac{\exp \left(-\left(t^{*}+1\right) \bar{\lambda}\right)}{1-\exp (-\bar{\lambda}) .}$

The proof now follows immediately from (22) for $\forall k \geq t^{*}+1$. For $k \leq t^{*}$, the result is obvious.

Now that we have identified a reasonable condition under which the uniform boundedness of $L_{k}(0)$ holds, we argue that $L_{k}$, as a solution of (16), is a continuous function of $\epsilon$ in a small neighborhood of $\epsilon=0$, and therefore, it will also be uniformly bounded for a sufficiently small $\epsilon$. This can be shown easily if the following assumption holds.

Assumption 4.2: $a_{L} c_{L}<1$, and there exists a positive real number $L_{\delta}$ such that

$$
a_{L} c_{L} L_{\delta}+b_{L}<L_{\delta}
$$

where there exist positive real numbers $a_{L}, b_{L}, c_{L}$ such that $\left\|\left|\left(\tilde{A}_{1}^{k}-L_{k}(0) \tilde{C}_{1}^{k}\right)^{-1}\left\|\left|<a_{L}, \|\right| L_{k}(0) \tilde{D}_{2}^{k}+L_{k}(0)\right.\right.\right.$ $\tilde{D}_{1}^{k} L_{k+1}(0)-\tilde{B}_{2}^{k}-\tilde{B}_{1}^{k} L_{k+1}(0) \mid \| \stackrel{b_{L}}{ }$ and $\| \tilde{C}_{2}^{k}+$
$\tilde{C}_{1}^{k} L_{k+1}(0) \mid \|<c_{L}$, where $\||\cdot|\|$ denotes a suitable matrix norm.

Notice that due to the finite number of discrete outputs $Y_{k+1}$ and the uniform boundedness of $L_{k}(0)$ easily guarantee the existence of $a_{L}, b_{L}, c_{L}$. The fact that $\left.\left(\partial L_{k} / \partial \epsilon\right)\right|_{\epsilon=0}$ satisfies the following recursive equation [easily derivable from (18)]

$$
\begin{aligned}
& \left.\left(\tilde{A}_{1}^{k}-L_{k}(0) \tilde{C}_{1}^{k}\right) \frac{\partial L_{k+1}}{\partial \epsilon}\right|_{\epsilon=0} \\
& =\left.\frac{\partial L_{k}}{\partial \epsilon}\right|_{\epsilon=0}\left(\tilde{C}_{2}^{k}+\tilde{C}_{1}^{k} L_{k+1}(0)\right)+L_{k}(0) \tilde{D}_{2}^{k} \\
& \quad+L_{k}(0) \tilde{D}_{1}^{k} L_{k+1}(0)-\tilde{B}_{2}^{k}-\tilde{B}_{1}^{k} L_{k+1}(0) \\
& \left.\frac{\partial L_{0}}{\partial \epsilon}\right|_{\epsilon=0}=0
\end{aligned}
$$

implies that Assumption 4.2 guarantees the uniform boundedness of $\left.\left(\partial L_{k} / \partial \epsilon\right)\right|_{\epsilon=0}$ such that $\left\|\left|\left(\partial L_{k} / \partial \epsilon\right)\right|_{\epsilon=0} \mid\right\|<L_{\delta}$. This allows us to use $L_{k}(0)$ as an $O(\epsilon)$ approximation to $L_{k}, \forall k$.

In addition, just as in [15], we make the following assumption, which has been justified in [15] and is seen to be satisfied through simulation studies.

Assumption 4.3: The evolution $z_{k+1}=z_{k}\left(\tilde{A}_{21}^{k} L_{k+1}+\tilde{A}_{22}^{k}\right)$, where $z_{k} \in \mathbb{R}^{n-N}$, has an exponentially stable solution.

Note that Assumption 4.3 guarantees that $\bar{\eta}_{k} \rightarrow 0$ asymptotically (using (15)). The rate of this decay is determined by the eigenvalues of $\tilde{A}_{21}^{k} L_{k+1}+\tilde{A}_{22}^{k}$ and how close they are to the origin. This also implies that there is a finite integer $k_{0}$ such that for $k>k_{0},\left|\bar{\eta}_{k}\right|$ is $O(\epsilon)$.

Using the above results [(14) and an unnormalized version of (15)], one can now write $O(\epsilon)$ approximations (for a sufficiently large $k$ ) to the unnormalized estimates $\zeta_{k}^{u}, \eta_{k}^{u}$ as

$$
\begin{aligned}
\tilde{\zeta}_{k+1}^{u} & =\tilde{\zeta}_{k}^{u}\left(\tilde{A}_{1}^{k}-L_{k}(0) C_{1}^{k}\right) \\
\tilde{\eta}_{k+1}^{u} & =-\tilde{\zeta}_{k+1}^{u} L_{k}(0) .
\end{aligned}
$$

We also assume that $\epsilon$ is small enough such that the normalization procedure [division by $\tilde{Z}_{k+1}$ in (15)] does not affect the order of approximation of the unnormalized quantity, that is, $\left(1 / Z_{k}\right)=\left(1 / \tilde{Z}_{k}\right)+O(\epsilon)$ for some $k \geq k_{1}$, where $\tilde{Z}_{k}=\tilde{\zeta}_{k}^{u} 1_{N}$.

The above discussion may be summarized in the form of the following theorem, establishing our main result on reducedorder approximations to the filtered estimates $\zeta_{k}, \eta_{k}$ :

Theorem 1: Suppose Assumptions 2.1, 4.1, 4.2, and 4.3 hold. In addition, suppose there exists an $\bar{L}>0$ such that the inequality (22) holds. Then, there exists a large enough but finite $k_{2}$ such that for $k \geq k_{2}$, an $O(\epsilon)$ approximation for $\zeta_{k+1}$ denoted by $\tilde{\zeta}_{k+1}$ (and the unnormalized version by $\tilde{\zeta}_{k+1}^{u}$ ) can be obtained recursively by the following two steps:

$$
\begin{aligned}
& \tilde{\zeta}_{k+1}^{u}=\tilde{\zeta}_{k}\left[\tilde{A}_{1}^{k}-L_{k}(0) \tilde{C}_{1}^{k}\right], \quad \tilde{\zeta}_{k_{2}-1}=\zeta_{k_{2}-1} \\
& \tilde{\zeta}_{k+1}=\frac{1}{\left[\tilde{\zeta}_{k+1}^{u} 1_{N}\right]} \tilde{\zeta}_{k+1}^{u} \cdot
\end{aligned}
$$

Similarly, an $O(\epsilon)$ approximation for $\eta_{k}$ (for $k \geq k_{2}$ ) is given by

$$
\tilde{\eta}_{k}=-\tilde{\zeta}_{k} L_{k}(0) \quad \tilde{\eta}_{k_{2}-1}=\eta_{k_{2}-1}
$$


TABLE I

TABle of Number of COMPUTATIONS PeR TIME InSTANT FOR EXACT AND APPROXimate $O(\epsilon)$ AgGregate Filtering

\begin{tabular}{c|c|c}
\hline No: of computations & Exact aggregate & $O(\epsilon)$ aggregate \\
\hline Addition & $n^{2}-1$ & $2 N^{2}+\sum_{i=1}^{N} n_{i}^{2}-n-1$ \\
\hline Multiplication & $n^{2}$ & $\sum_{i=1}^{N} n_{i}^{2}+n N-N$ \\
\hline Division & $N$ & $2 N$ \\
\hline
\end{tabular}

Similarly, an $O(\epsilon)$ approximation to $\alpha_{k}$ (the full-order normalized filtered estimate) is given by

$$
\tilde{\alpha}_{k}=\tilde{\zeta}_{k} V_{1}+\tilde{\eta}_{k} V_{2}
$$

We now provide Table I, which compares the numbers of additions, multiplications, and divisions per discrete time instant in computing the exact aggregate filtered estimates $\left(\zeta_{k}\right)$ and the corresponding $O(\epsilon)$ approximation $\left(\tilde{\zeta}_{k}\right.$, as given by Theorem 1$)$.

It is clear that there will be substantial savings in computations when the Markov chain has a reasonable number of superstates of small individual dimensions. For example, for $n=200$ with $N=40, n_{i}=5, \forall i$, we have the number of additions, multiplications, and divisions for the exact aggregate filtering as 39999, 40000, and 40, respectively. The corresponding numbers for approximate aggregate filtering are 3999, 8960, and 80, respectively. Similar computational savings can be demonstrated for full-order filtering as well.

\section{Simulation StUdies}

In this section, we present some simulation studies to demonstrate the performance of our reduced-complexity filter. The performance measures used are average approximation error in aggregate filtering and full-order filtering. The average aggregate filtering error is given by $E\left[\left\|\zeta_{k}-\widetilde{\zeta}_{k}\right\|^{2}\right]$, which (from ergodicity assumptions) is estimated by the average approximation error $\lim _{T \rightarrow \infty}(1 / T) \sum_{k=1}^{T}\left\|\zeta_{k}-\tilde{\zeta}_{k}\right\|^{2}$. Similarly, the average full-order approximation error is given by $E\left[\left\|\alpha_{k}-\tilde{\alpha}_{k}\right\|^{2}\right]$, which in turn is estimated by $\lim _{T \rightarrow \infty}(1 / T) \sum_{k=1}^{T}\left\|\alpha_{k}-\tilde{\alpha}_{k}\right\|^{2}$.
For our simulations, the choice for $T$ is 100000 . We also average our results over ten simulations.

As in [15], we perform our simulations with an eight-state ( $n=8, N=3$ ) nearly completely decomposable Markov chain with a transition probability matrix specified by (29), shown at the bottom of the page. The discrete observation (or measurement) set contains three possible outputs where the state-to-observation probability matrix is given by

$C=\left[\begin{array}{cccccccc}0.2 & 0.15 & 0.5 & 0.3 & 0.2 & 0.8 & 0.75 & 0.25 \\ 0.26 & 0.7 & 0.25 & 0.45 & 0.47 & 0.16 & 0.15 & 0.68 \\ 0.54 & 0.15 & 0.25 & 0.25 & 0.33 & 0.04 & 0.1 & 0.07\end{array}\right]$

Clearly, the choice for $W_{1}, W_{2}, V_{1}$, and $V_{2}$ here is the same as in [15] and is not repeated here.

Remark 3: Note that in this example, $C$ does not have any special structure as in [15]. The only restriction on $C$ is that all elements are positive. Therefore, the results of this paper are applicable to a very general class of state to observation probability matrices.

It was shown in [15] that the various aggregation methods that exist in the literature such as Courtois' method and Khalil's method [10] can be extended to obtain ad hoc approximate aggregate filters that often result in inexplicably large approximation errors, especially in the case of a $C$ matrix, which cannot be readily aggregated unlike a block-structured $C$, as in [15]. Therefore, here, we show no comparisons with other aggregation methods for our approximate aggregate filter. Note also that the full-order approximate filtering scheme is unique to our approach and cannot be obtained by extending any of the aggregation methods.

The $O(\epsilon)$ nature of the approximation errors is illustrated in Table II, which shows the average approximation error for both the approximate aggregate filter and the full-order approximate filter, as obtained by our approach for various values of $\epsilon$ ranging from 0.001 to 0.1 .

Various numerical issues that can arise here in implementing our algorithm regarding stability of $L_{k}(0)$ or how large $\epsilon$ can

$$
\begin{aligned}
A & =\left[\begin{array}{cccccccc}
-0.45 & 0.25 & 0.20 & 0 & 0 & 0 & 0 & 0 \\
0.2 & -0.45 & 0.25 & 0 & 0 & 0 & 0 & 0 \\
0.24 & 0.35 & -0.59 & 0 & 0 & 0 & 0 & 0 \\
0 & 0 & 0 & -0.3 & 0.3 & 0 & 0 & 0 \\
0 & 0 & 0 & 0.2 & -0.2 & 0 & 0 & 0 \\
0 & 0 & 0 & 0 & 0 & -0.40 & 0.25 & 0.15 \\
0 & 0 & 0 & 0 & 0 & 0.20 & -0.45 & 0.25 \\
0 & 0 & 0 & 0 & 0 & 0.25 & 0.15 & -0.40
\end{array}\right] \\
B & =\left[\begin{array}{ccccccccc}
0.1 & 0.1 & -1.0 & 0.6 & 0.05 & 0.05 & 0.05 & 0.05 \\
0.05 & 0.05 & -0.9 & 0.5 & 0.05 & 0.05 & 0.1 & 0.1 \\
0.01 & 0.01 & -0.4 & 0.2 & 0.05 & 0.05 & 0.04 & 0.04 \\
0.02 & 0.42 & 0.01 & 0.01 & -0.61 & 0.025 & 0.1 & 0.025 \\
0.45 & 0.01 & 0.4 & -1.0 & 0.01 & 0.1 & 0.01 & 0.02 \\
0.01 & 0.05 & 0.01 & 0.01 & 0.05 & 0.01 & -0.15 & 0.01 \\
0.03 & 0.01 & 0.03 & 0.04 & 0.01 & 0.01 & 0.01 & -0.14 \\
0.01 & 0.05 & 0.01 & 0.01 & 0.05 & -0.16 & 0.01 & 0.02
\end{array}\right]
\end{aligned}
$$


TABLE II

Table of Average ApProXimation ERror IN AgGregate AND FULL-ORDER FILTERING

\begin{tabular}{c|c|c}
\hline \multirow{2}{*}{$\epsilon$} & \multicolumn{2}{|c}{ Average approximation error } \\
\cline { 2 - 3 } & Aggregate filter & Full-order filter \\
\hline 0.001 & $3.751 \times 10^{-6}$ & $2.7483 \times 10^{-6}$ \\
\hline 0.005 & $5.0458 \times 10^{-6}$ & $6.7955 \times 10^{-6}$ \\
\hline 0.008 & $9.526 \times 10^{-6}$ & $1.562 \times 10^{-5}$ \\
\hline 0.01 & $1.5132 \times 10^{-5}$ & $2.2048 \times 10^{-5}$ \\
\hline 0.03 & $2.5031 \times 10^{-5}$ & $1.0583 \times 10^{-4}$ \\
\hline 0.05 & $4.9878 \times 10^{-5}$ & $2.5571 \times 10^{-4}$ \\
\hline 0.08 & $1.1945 \times 10^{-4}$ & $6.5083 \times 10^{-4}$ \\
\hline 0.1 & $1.73252 \times 10^{-4}$ & $1.02574 \times 10^{-3}$ \\
\hline
\end{tabular}

be, etc., will not be specifically addressed in this work as the corresponding discussions are very similar to those in [15]. One note should be added that if, for a particular $k, L_{k}(0)$ cannot be computed due to the matrix $\left(\tilde{A}_{1}^{k}-L_{k}(0) \tilde{C}_{1}^{k}\right)$ being singular, one should discard that observation and reinitialize the algorithm. We know that due to the exponential forgetting property of HMM filters (see [19]) that such interruptions will not have a significant effect in the asymptotic performance of the algorithm.

\section{CONCLUSION}

In this paper, we addressed the problem of reduced-complexity state estimation for hidden Markov models with nearly completely decomposable Markov chains. This research addresses the problem for a general state to output transition matrix structure rather than the special structure studied earlier in [15]. It is shown that under natural irreducibility assumptions on the underlying Markov chain and for a sufficiently small $\epsilon$, one can obtain $O(\epsilon)$ approximations to the aggregate as well as the full-order filter with reduced-complexity computations. The computational savings are substantial when the underlying Markov chain has blocks with small individual dimensions. Exact computational complexity of our algorithm is provided, along with some simulation studies demonstrating the performance guarantee on the accuracy of the algorithm. In addition, one can apply our algorithm to obtain reduced-complexity filtered estimates for HMMs with continuous-range (real valued) outputs as well. In fact, simulations demonstrate that the algorithm works quite well in this case, but many of the rigorous proofs (such as for Lemma 4.1) need to be carefully worked out. This is part of our ongoing work.

\section{REFERENCES}

[1] F. Delebecque and J. P. Quadrat, "Optimal control of Markov chains admitting strong and weak interactions," Automatica, vol. 17, pp. 281-296, 1981.

[2] R. G. Phillips and P. V. Kokotovic, "A singular perturbation approach to modeling and control of Markov chains," IEEE Trans. Automat. Contr., vol. AC-26, pp. 1087-1094, 1981.

[3] V. Krishnamurthy, "Adaptive estimation of hidden nearly completely decomposable Markov chains with applications in blind equalization," Int. J. Adaptive Contr. Signal Process., vol. 8, pp. 237-260, Aug. 1994.
[4] L. White, R. Mahony, and G. Brushe, "Lumpable hidden Markov models-Model reduction and reduced complexity filtering," IEEE Trans. Automat. Contr., vol. 45, pp. 2297-2306, Dec. 2000.

[5] H. A. Simon and A. Ando, "Aggregation of variables in dynamic systems," Econometrica, vol. 29, pp. 111-138, 1961.

[6] P. J. Courtois, Decomposability Queueing and Computer System Applications. New York: Academic, 1977.

[7] G. Yin and Q. Zhang, Continuous-Time Markov Chains and Applications: A Singular Perturbation Approach. New York: Springer-Verlag, 1998.

[8] D. Tse, R. Gallager, and J. Tsitsiklis, "Statistical multiplexing of multiple time-scale Markov streams," IEEE J. Select. Areas Commun., vol. 13, pp. 1028-1038, Nov./Dec. 1995.

[9] C. D. Meyer, "Stochastic complementation, uncoupled Markov chains, and the theory of nearly reducible systems," SIAM Rev., vol. 31, pp. 240-272, June 1989.

[10] R. W. Aldhaheri and H. K. Khalil, "Aggregation of the policy iteration method for nearly completely decomposable Markov chains," IEEE Trans. Automat. Contr., vol. 36, pp. 178-187, Feb. 1991.

[11] M. Coderch, A. S. Willsky, S. S. Sastry, and D. A. Casatanon, "Hierarchical aggregation of singularly perturbed finite state Markov processes," Stochastics, vol. 8, pp. 259-289, 1983.

[12] J. R. Rohlicek and A. S. Willsky, "The reduction of perturbed Markov generators: An algorithm exposing the role of transient states," J. Assoc. Comput. Machinery, vol. 35, pp. 675-696, 1988.

[13] F. Delebecque, J. P. Quadrat, and P. V. Kokotovic, "A unified view of aggregation and coherency in networks and Markov chains," Int. J. Contr., vol. 40, pp. 939-952, 1984.

[14] G. Yin and S. Dey, "Weak convergence of hybrid filtering problems involving nearly completely decomposable hidden markov chains," SIAM J. Contr. Optimization, vol. 41, no. 8, pp. 1820-1842, 2003.

[15] S. Dey, "Reduced-complexity filtering for partially observed nearly completely decomposable Markov chains," IEEE Trans. Signal Processing, vol. 48, pp. 3334-3344, Dec. 2000.

[16] L. Shue and S. Dey, "Complexity reduction in fixed-lag smoothing of hidden markov models," IEEE Trans. Signal Processing, vol. 50, pp. 1124-1132, May 2002.

[17] L. R. Rabiner, "A tutorial on hidden Markov models and selected applications in speech recognition," Proc. IEEE, vol. 77, pp. 257-285, Feb. 1989.

[18] P. Kokotovic, H. K. Khalil, and J. O'Reilly, Singular Perturbation Methods in Control: Analysis and Design. New York: Academic, 1986.

[19] L. Shue, B. D. O. Anderson, and S. Dey, "Exponential stability of filters and smoothers for hidden Markov models," IEEE Trans. Signal Processing, vol. 46, pp. 2180-2194, Aug. 1998.

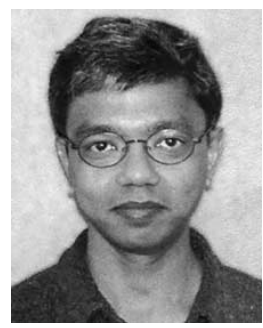

Subhrakanti Dey (M'96) was born in Calcutta, India, in 1968. He received the B.Tech. and M.Tech. degrees from the Departent of Electronics and Electrical Communication Engineering, Indian Institute of Technology, Kharagpur, India, in 1991 and 1993, respectively, and the Ph.D. degree from the Department of Systems Engineering, Research School of Information Sciences and Engineering, Australian National University, Canberra, Australia, in 1996.

He has been a Senior Lecturer with the Department of Electrical and Electronic Engineering, University of Melbourne, Parkville, Australia, since February 2000. From September 1995 to September 1997 and September 1998 to February 2000, he was a postdoctoral Research Fellow with the Department of Systems Engineering, Australian National University. From September 1997 to September 1998, he was a post-doctoral Research Associate with the Institute for Systems Research, University of Maryland, College Park. His current research interests include signal processing for telecommunications, wireless communications and networks, performance analysis of communication networks, stochastic and adaptive estimation and control, and statistical and adaptive signal processing. 


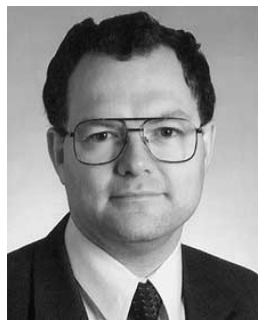

Iven Mareels (F'01) was born in Aalst, Belgium, on August 11, 1959. He received the B.Sc. degree in electromechanical engineering from Gent University, Gent, Belgium, in 1982 and the Ph.D. degree in systems engineering from the Australian National University, Canberra, Australia, in 1987.

$\mathrm{He}$ has been Professor with the Department of Electrical and Electronic Engineering, the University of Melbourne, Parkville, Australia, where he has held the Chair of Electrical and Electronic Engineering since 1996. Previously, he was a Reader at the Australian National University (from 1990 to 1996), a lecturer at the University of Newcastle, Callaghan, Australia (from 1988 to 1990), and the University of Gent (from 1986 to 1988). He has extensive experience in consulting for both industry and government. He has strong interests in education and, in particular, is experimenting with multimedia techniques in the undergraduate engineering curriculum. He has taught a broad range of subjects in both mechanical and electrical engineering curricula. His research interests are in adaptive and learning systems, nonlinear control, and modeling. At present, he has strong research interests in modeling and controlling of large-scale networked systems and environmental systems, in particular with applications in natural resource management. He has co-authored three research monographs, about 100 journal papers, and more than 150 conference publications and received several awards for his publications.

Dr. Mareels was a recipient of the Vice-Chancellor's Award for Excellence in Teaching in 1994. He is a co-editor in chief, together with Prof. A. Antoulas, of the International Journal Systems and Control Letters. He is Fellow of the Academy of Technological Sciences and Engineering, Australia, a member of the Society for Industrial and Applied Mathematics, and a Fellow of the Institute of Engineers Australia. He is a member of the Asian Control Professors Association, Chairman of the Education Committee of the latter, and the Vice Chair of the Steering Committee for the Asian Control Conference. He is a member of the Board of Governors of the IEEE Control Systems Society. He is registered with the Institute of Engineers Australia as a professional engineer. 